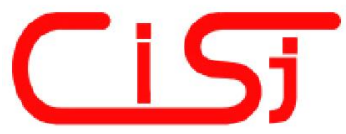

computing@computingonline.net www.computingonline.net
Print ISSN 1727-6209

On-line ISSN 2312-5381

International Journal of Bomputing

\title{
OBSERVATIONS-BASED COMPUTATIONAL ANALYTICS ON LOCAL CLIMATE DYNAMICS. PART 3: FORECASTING
}

\author{
Yury Kolokolov, Anna Monovskaya \\ Orel State University, Komsomolskaya str. 95, 302026, Orel, Russia, www.oreluniver.ru \\ 2kolo@mail.ru, anna.monovskaya@mail.ru
}

\begin{abstract}
Computational decision making is discussed in application to seasonal temperature forecasts taking into account inevitable nonlinear nature of local climate systems and deficiency of data on reliable observations. We focus on temperature extremes in terms of daily means and first involve the alternative conceptual model of local climate dynamics (the model of hysteresis regulation with double synchronization, so-called HDS-model) into such analytics. Recent years the HDS-model is describing successfully abnormal interannual temperature variability, on the basis of which it becomes potentially possible to extend forecasts of local daily means up to more than 1 year in future. In this connection the novel method of bifurcation traps is proposed, realized and tested. Results of processing the time series of temperature observations on daily mean surface air temperature illustrate peculiarities of this method in comparison with the traditional viewpoint on the forecasts. We believe that the discussion could be interesting in science and practice in order to increase the confidence of estimations on coming climate changes. Copyright $\mathbb{C}$ Research Institute for Intelligent Computer Systems, 2017. All rights reserved.
\end{abstract}

Keywords: Nonlinear dynamics forecasting, HDS-model, seasonal forecast, bifurcation analysis, likely periodicity, annual temperature variation, annual warming-cooling cycle, temperature extreme, local climate changes.

\section{INTRODUCTION}

Research in the field of the present-day climatology seems to be impossible to realize without attracting information technologies starting with local meteorological observations $[1,2,3]$ towards analytics concerning regional $[4,5,6]$ and global $[7,8,9]$ climate processes and practiceoriented services $[10,11,12]$. Among great many of the current tasks, forecasting of dynamics evolution of local climate systems seems to be one of the main challenges, especially, in seasonal terms $[13,14,15]$. Such particular attention occurs since any practical activity depends on weather and climate effects in more or less extent. At the same time, local climate systems can demonstrate too high variability in their behaviors $[6,8,16,17]$. So, the more coming local climate changes become clear, the more chances will be to adapt activities to them.

Historically, the habitual statements of weather forecasting and climate prediction not include some practice-oriented contexts which are up-to-date $[13,15,18]$. In particular, tools of weather forecasting are not enough in order to cover the seasonal horizon with daily resolution; tools of climate prediction are not enough to consider seasonal details in a decadal scale. So, the existing methods of estimations towards future do not suppose to consider seasonal forecasting with local daily means for extended time horizons (more than 1 year). The corresponding remarks to the problem statement we introduce in section 2. However, it is only one of third problems which are fundamental obstacles to realize the practice-oriented local forecasting discussed in the paper. The rest two problems concern the following well-known questions: how to get enough reliable data?; and how to distinguish reasonably qualitative and quantitative changes in dynamics evolution?

Concerning the data, let us emphasize two items: too short historical horizons (no more than 150 years for typical climate estimations in several decades); uniqueness of the analyzed nonlinear system (there are not abilities to get a lot of experimental data on Earth dynamics as it could be for an experimental setup). As a result, really irreparable data shortage remains. Concerning climate changes, let us emphasize the physical features of local climate system regulation $[19,20,21]$ : natural feedbacks with quantization in time and in level, as a result of which hysteresis phenomena, esential interannual variability and bimodality of statistical distributions inevitably appear.

These questions are caused by integral features of nonlinear phenomena observed in local climate 
systems [6] and should be encapsulated in a conceptual model used while weather and climate analytics. Traditionally, only one normal periodiclike annual behavior is built-in to the climate model $[19,20,22]$, and such viewpoint can meets troubles while its use $[16,17,18]$. The conceptual model of the hysteresis regulation with double synchronization (so-called HDS-model) has opened a way to the bifurcation analysis of the local dynamics by the specilized processing the meteorological observations and proposed an alternative viewpoint on normal behaviors [21].

Thus, at present, there is a variant on how to distinguish reasonably qualitative and quantitative changes. Also, the HDS-model allows to derive some constituents of regional (i.e. external) impacts on a local climate system by analysis of its temperature observations, that could be promising to reconstruct missing data $[23,24]$. Since the HDSmodel presents local behaviors in terms of nonlinear dynamics, then we include brief comments to specify the differences of this viewpoint relatively the discussed subject (section 3). Next, we present the novel method of forecasting "traps" to evolution of maximums and minimums of local daily mean temperature (section 4) and results of its testing (section 5). Main outcomes and future outlook are summarized in section 6 .

\section{PROBLEM STATEMENT}

Since the discussion relates to the interdisciplinary field, let us make the necessary remarks. The notion of "forecasting" means that the necessary information from the future should be known at present. Let time interval from such future to the present will be denoted as an "advance time". In general, there are two variants on how it could be achieved for each time scale: by a model which should generate desirable solutions; by processing the data of meteorological observations and/or paleo-data to make desirable estimations towards future. In particular, forecasts of annual temperature maximums and minimums touch the weather, seasonal and climate scales more or less.

For example, confidence of weather forecasting could be preferable, however, such confidence is accompanied by the advance time of no more than 710 days long that remains inevitably restricted by the "butterfly effect" $[25,26]$. And the weather models become more and more deprived of the support provided by climate norms due to the accelerated climate changes $[27,28,29]$. Interpolations of climate tendencies made on the basis of already realized temperature observations relate to desirable advance time of several years long; however, such interpolations remains quite cautious [19, 22, 29].
Nevertheless, we involve this direction into discussion due to it supposes certain statistical confidence.

Decadal predictions made by the advanced climate models relate to global or regional mean temperatures without necessary details, for example $[5,6,19,22]$. Seasonal models are specialized for regional estimations formulated in terms of "above average", "near average" or "below average" concerning monthly and even seasonal means with confidence about 40-80\%. Usually, the corresponding advance time is from month to three months long [30, 31, 32]; in some cases, seasonal forecasts are extended to 6 months [33]; and only experimental seasonal forecasts allow to cover 1 year [34] that becomes consistent with the aim formulated in 2012 by [35]. In this context the discussed extension of the advanced time under increase in daily confidence represents a novel problem statement for seasonal forecasting.

\section{FUNDAMENTALS}

Any method of forecasting is based on the corresponding conceptual model which describes the spectrum of events and phenomena supposed to be forecasted. In particular, the conceptual model of annual temperature variation (ATV-model) remains the basic one in order to determine climate norms, to analyze climate changes and to assess climate risks, for example $[19,22,36]$. The ATV-model describes the observed variability by one averaged annual behavior, where deviations from mean values excused by chaotic nature and are statistically determined. Such uni-behavior model has serious disadvantages which have been discussed $[16,17$, 37]. Let us focus on the aspects which are crucial for the discussion, and let us consider a linearized view, where maximums and minimums become the main elements to determine ATV-model.

Each such element is calculated as average temperature $(\mathrm{T})$ over 30-year window with daily, or monthly, or seasonal resolution (Fig. 1, a, b, c correspondingly); and differences between these averaging scales can be essential (several tens percents) for annual extremes $\left(\mathrm{T}_{\min }\right.$ and $\left.\mathrm{T}_{\max }\right)$. Also, the averaging creates significant difficulties for sewing weather and climate notions $[18,27]$ and creates statistical misses (for example, see below Fig. 4a) which indicate formally heterogeneity of the analyzed data $[16,17,21]$. So, these circumstances lead to the necessity of additional research concerning conditions on how to provide correctness and truthfulness of the statistical analysis and, correspondingly, trend estimations which really remain the basic tool to realize successful forecasting. 


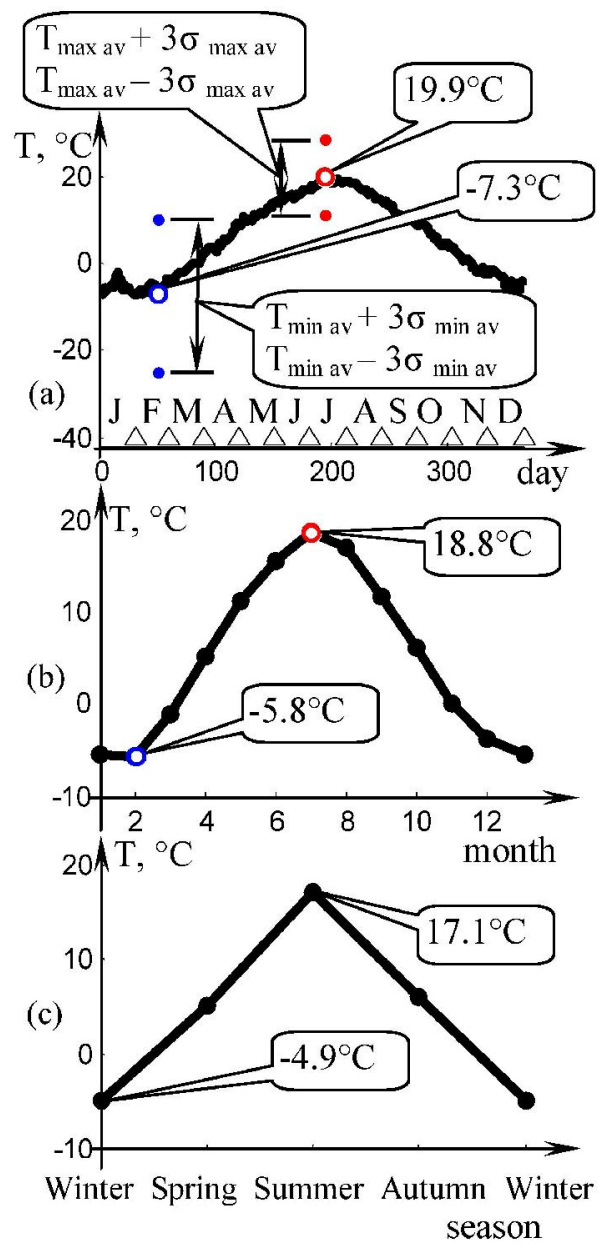

Fig. 1 -Difference in temperature extremes averaged over 1981-2010 years with daily (a), monthly (b) and seasonal (c) resolution (non-average maximum and minimum are equal to $30.1^{\circ} \mathrm{C}$ and $-33.6^{\circ} \mathrm{C}$ correspondingly).

The HDS-model represents a dynamical system with a variable structure and is recently used as an alternative model to determine climate norms, to analyze climate changes and to assess climate risks. Description of the mathematical view of HDS-model (including the hysteresis built-in regulator), peculiarities and regularities of HDS-dynamics, building bifurcation diagrams and reconstruction of HDS-model parameters were presented in detail in $[18,21,27]$. The main differences of this model from the ATV-model can be covered by the following three items: multi-behavior; relative scales in time and in temperature; variety of local evolutional scenarios (including variety of time windows, where heterogeneity of dynamics is kept). Let us specify briefly these items.

From the HDS-model viewpoint, any local climate system can choice one of three kinds of behaviors each year (Fig. 2, a), where $L$-behavior is comparatively "warm", $R$-behavior is comparatively "cold", and $C$-behavior is comparatively neutral (warmer than $R$-behavior and colder than $L$ behavior). These behaviors are characterized by 1 - year periodicity but different annual patterns concerning the vertical centerline (dash-dot red lines in Fig. 2, a). The notion of "comparatively" appears due to each ensemble of $R$-, $L$-, $C$-behaviors (Fig. 2, b) is encapsulated in the context of the moving temperature hysteresis (Fig. 2, c) determined by unique local $\mathrm{H}$-limit, $\mathrm{T}_{\text {ref }}$-horizontal centerline and two synchronization surfaces $\left(F_{1}\right.$ and $\left.F_{2}\right)$ with a half year shift. The last was discussed in detail in [23, 24]. So, in the case of the ATV-model, a built-in regulator is absent and both time and temperature coordinates are fixed and adjusted (Fig. 1, a, b, c). At the same time, there are two fact, in accordance to which this uni-behavior model remains the most widespread and convenient for the habitual thinking till now. First, $C$-behavior looks like ATV-model behavior most of all; second, $C$-behavior is dominant in local climate dynamics [6, 21, 27].
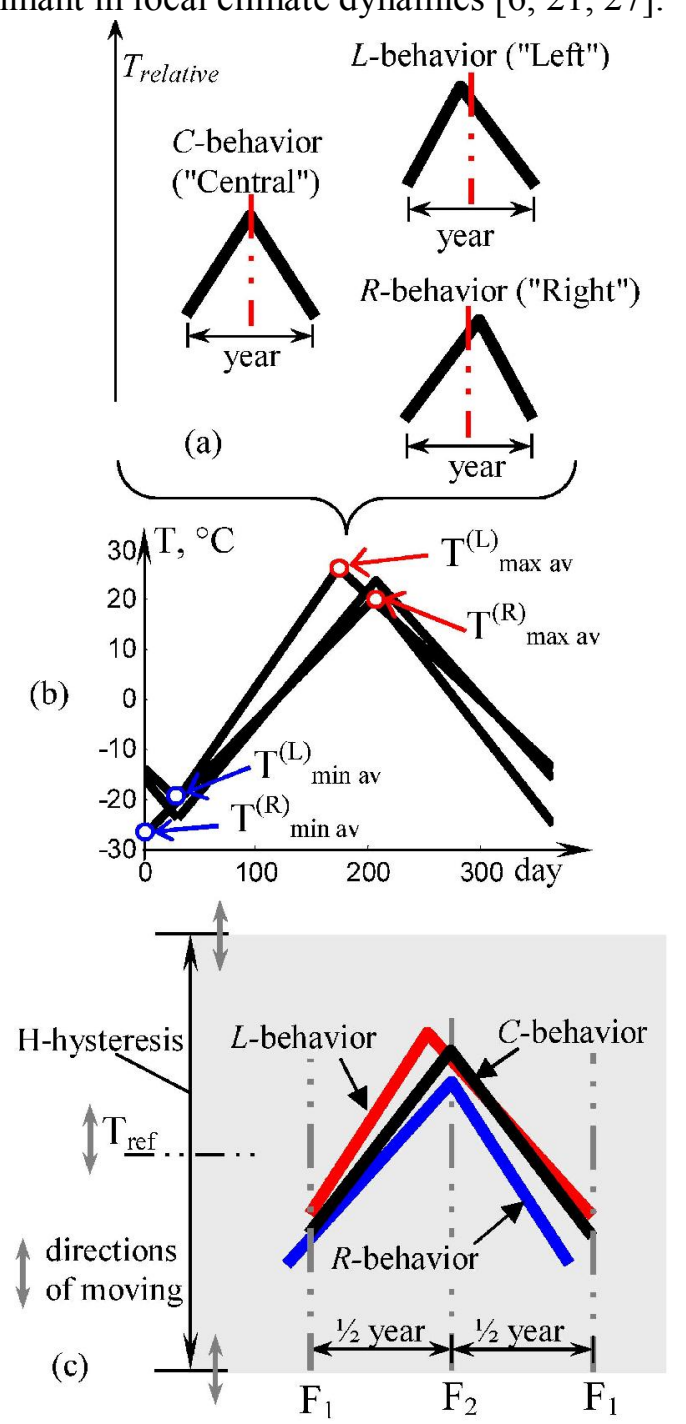

Fig. 2 - Scheme to $R$-, $L$-, $C$-behaviors (a); an example of one of $R L C$-ensembles over 1981-2010 years (b) in comparison with annual cycles in Fig.1; scheme to $R L C$-ensemble placed in the relative coordinate (c).

Strictly speaking, the qualitative changes in local climate dynamics should be identified by the 
bifurcation analysis [21]. Results of such analysis demonstrate the phenomenon of "likely periodicity" [27]: each sunspot extremum provides the possibility to change qualitatively the dynamics of local climate systems, but each local climate system can realize such possibility or can ignore it. We pay attention to this result due to the practical viewpoint. Namely, the bifurcation analysis is realized by tens of specialized numerical computer procedures which are integrated with built-in interactive expert choices and approvals. So, any reasonable variant on how to simplify and/or formalize these procedure seems to be interesting. The "likely periodicity" excludes quite expensive analytics by the assumption on 11year window sliding in 1 year. In this case, of course $R, L, C$-ensembles become reconstructed in outline, however tendencies of dynamics evolution can be accepted for pilot testing and we first use this simplification (see below Fig. 5, b).

\section{METHOD OF BIFURCATION TRAPS}

Let us focus on the novelty of the proposed method and IT-tools to realize it. We started from the HDS-model to decompose intricate dynamics of a local climate system into a set of behaviors $(R-, L-$, $C$-behaviors illustrated in Fig. 2, a, b, c). In this connection each $T$-time series is divided into three corresponding sets. So, evolution of the behaviors can be statistically analyzed due to each of them represents a homogeneous set. With this purpose, we use the approach on how to reconstruct the $R L C$ ensemble presented in details in [27]. For example (Fig. 3, a), if 1973-year is associated with $L$ behavior and 1974-year is associated with $R$ behavior, then the first $T$-time series fragment belongs to the set of $L$-behavior and the second one belongs to the set of $R$-behavior. The proposed method of forecasting is focused on how to provide novel abilities of forecasts on the basis of such disassembling? And we restrict the research by annual maximums and minimums in daily terms (Fig. 1, a).

What is the origin of the novel abilities? When a local climate norm is built in accordance with the ATV-model (Fig. 1, a), then both average maximum $\left(\mathrm{T}_{\max \text { av }}\right)$ and average minimum $\left(\mathrm{T}_{\min \text { av }}\right)$ are estimated with the corresponding standard deviations $\left(\sigma_{\max }\right.$ av and $\sigma_{\min }$ av $)$. However, from the HDS-model, these characteristics represent resultant notions, where ensemble of $R-, L-, C$-behaviors should be considered in the context of the moving temperature hysteresis (Fig. 2, c). It means, that $\mathrm{T}_{\max }$ av is a results of a superposition of $\mathrm{T}^{(\mathrm{L})}{ }_{\max }$ av, $\mathrm{T}^{(\mathrm{C})}{ }_{\max }$ av, and $\mathrm{T}^{(\mathrm{R})}{ }_{\max }$ av, where indexes in brackets denote the corresponding kind of the behavior (Fig. 3, a). The same situation occurs in the cases of $T_{\min \text { av }}, \sigma_{\max }$ av , and $\sigma_{\min \text { av }}$
(Fig. 3, a, b), where each average value is linked with one standard deviation of the same kind (denoted by two-sided arrows). For example, let us consider a fragment of $T$-time series about 1974 year (Fig. 3, b): the annual temperature maximum (denoted by a red circle) is the same for ATV-model $\left(\mathrm{T}_{\max }\right)$ and for HDS-model $\left(\mathrm{T}^{(\mathrm{R})}{ }_{\max }\right)$, however $\mathrm{T}^{(\mathrm{R})}{ }_{\max }-$ value belongs to the set of $R$-behavior only; at the same time, the annual temperature minimums are significantly different (denoted by a green circle for ATV-model and by a blue circle for HDS-model), and $\mathrm{T}^{(\mathrm{R})}{ }_{\min }$-value belongs to the set of $R$-behavior only. Correspondingly, the first main procedure of the proposed method (denoted by bold letters in Fig. 4, a) is aimed at calculation of 12 basic characteristics of HDS-model encapsulated within the braces in Fig. 3, a, b.

$$
T_{\text {max av }}\left\{\begin{array}{lll}
\mathrm{T}^{(\mathrm{L})}{ }_{\text {max av }} & \leftrightarrow & \sigma^{(\mathrm{L})}{ }_{\text {max av }} \\
\mathrm{T}^{(\mathrm{C})} \\
\mathrm{T}_{\text {max av }}^{(\mathrm{R})}{ }_{\text {max av }} \leftrightarrow & \leftrightarrow \sigma^{(\mathrm{C})}{ }_{\text {max av }}^{(\mathrm{R})}{ }_{\text {max av }}
\end{array}\right\} \sigma_{\text {max av }}
$$

(a)

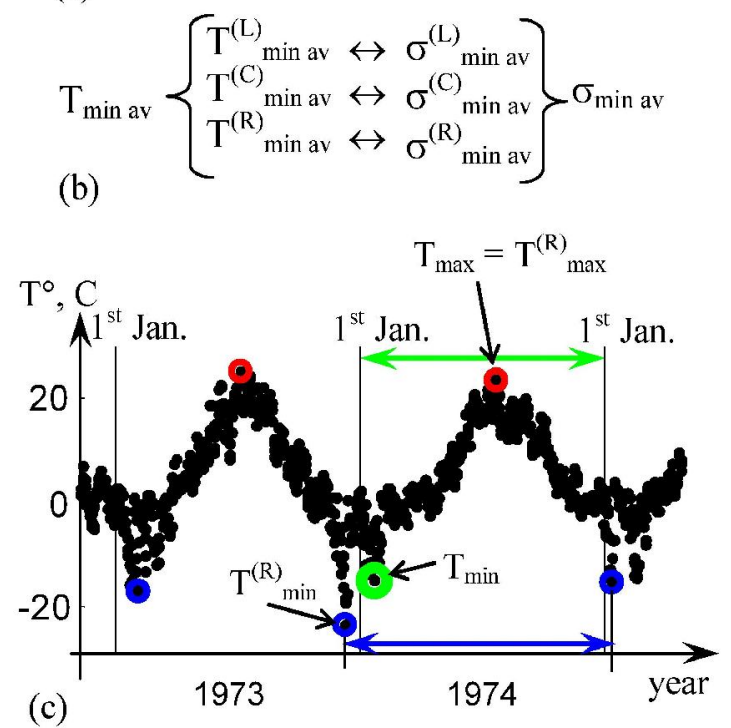

Fig. 3 - Differences between ATV- and HDS-models illustrated by a fragment of T-time series over 19731974 years (a) and ensembles of characteristics for the temperature maximum (b) and minimum (c).

The second main procedure (Fig. 4, a) is aimed at building the extremely possible temperature limits for each year, similar to upper and lower limits of the temperature maximum $\left(\mathrm{T}_{\max a v}+\sigma_{\max }\right.$ av and $\mathrm{T}_{\max }$ av $-\sigma_{\max \text { av }}$, Fig. 1, a) and the temperature minimum $\left(\mathrm{T}_{\text {min av }}+\sigma_{\text {min av }}\right.$ and $\mathrm{T}_{\min \text { av }}-\sigma_{\min \text { av }}$ Fig. 1, a) determined for ATV-model. Strictly speaking, we calculate all the possible combinations grounded on 12 basic characteristics mentioned before (Fig. 3, a, b), and then select the maximal and minimal values. Usually, the upper and lower limits are associated with $L$ - and $R$-behaviors (red and blue circles in Fig. 2, b correspondingly): $\mathrm{T}^{(\mathrm{L})}{ }_{\max }$ av and $\mathrm{T}^{(\mathrm{R})}{ }_{\max }$ av in the case of the temperature maximum; $\mathrm{T}^{(\mathrm{L})}{ }_{\min }$ av and 
$\mathrm{T}^{(\mathrm{R})}{ }_{\text {min av }}$ in the case of the temperature minimum. However, we check all the cases (including $C$ behavior) due to the following variant can appear sometimes: $C$-behavior can be very close to $L$ - or $R$ behavior and its standard deviation $\left(\sigma^{(\mathrm{C})}{ }_{\max }\right.$ av or $\sigma^{(\mathrm{C})}{ }_{\min }$ av) can even exceed the $L$ - or $R$-standard deviation. In other words, $C$-behavior, which is like ATV-model behavior most of all, is slightly used that illustrates the original difference between the proposed and traditional processing.

The third main procedure is aimed at decisionmaking on how to forecast the dynamics of the identified upper and lower temperature limits and how to estimate the accuracy of the forecasting in (year, T)-space in comparison with the traditional viewpoint. In this connection we introduce the notion of a "temperature trap" - a geometrical figure in (year, T)-space, into which all future events should hit without misses. Then, first, if even one miss occurs, then this forecast is false. Second, comparing results of several forecasts show the comparing sizes of margins within the corresponding traps: the smaller margin is, the better forecast is. These two conditions shift the accent from probabilistic estimations with more or less confidence to estimations of the margins within the traps under $100 \%$ probability. Building the temperature traps (Fig. 4, a) includes several steps (described by the next section), and, finally, the rectangular traps are built towards future $\left(\mathrm{T}_{\max }\right.$ and $\mathrm{T}_{\text {min }}$-traps denoted by red and blue dotted contours in Fig. 5, a, b) to caught future events (black circles).

Software to realize and test the proposed method in comparison with the traditional statistical estimations is designed on MatLAB-platform. The research follows to the main cycle shown in Fig. 4, and is integrated into the analytical system intended for estimating the severity level of imminent danger caused by local climate changes. This specilized system provides realization of the novel interrelated computations in accordance with HDS-model conceptions. These computations are logically divided within six functional modules (Fig. 4, b): data collection and translation; data banks; dynamics modeling (computer-based simulations); time series analysis (processing the observations); dynamics reconstruction, where measured and simulated results are synthesized; risk assessment. The procedures of the earlier designed software are denoted by gray in Fig. 4, a, the procedures of the new designed software are denoted by white in Fig. 4, a. Data transfer between the modules is realized by means of built-in SAVE/LOAD functions and MAT-files created by the corresponding executed programs. Integration is specified by data flows (Fig. 4, c), where $T$-time series and RLC-encembles are transferred for testing from the modules of data banks and dynamics reconstruction correspondingly.

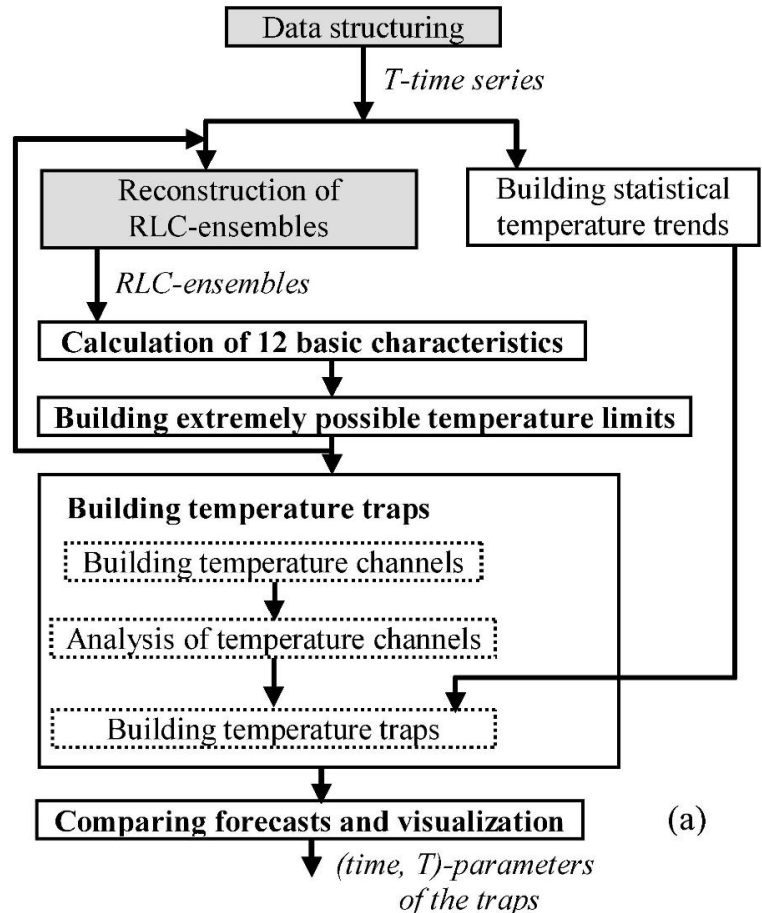

of the traps
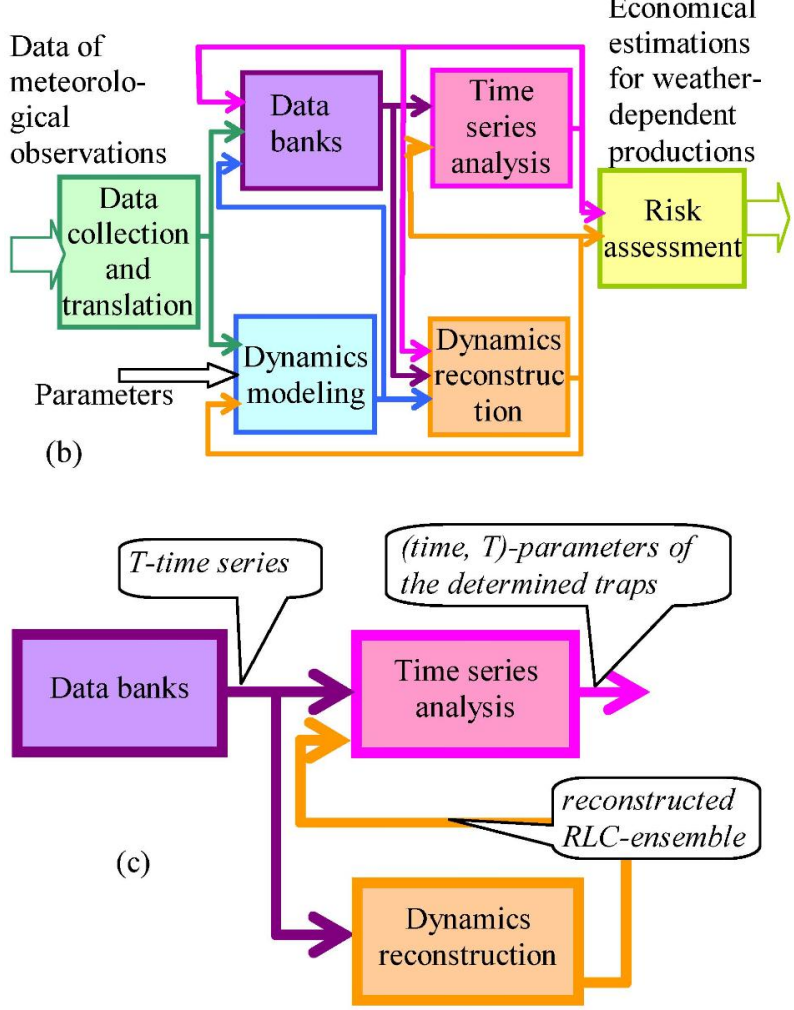

Fig. 4 - The main cycle of testing (a), the logical scheme of the analytical system (a), involved data flows (b) to realize the testing.

\section{EXAMPLE OF TESTING}

The method of forecasting traps were tested by processing the open-access data of the meteorological observation on daily mean surface air temperature provided by Russian Research Institute of Hydrometeorological Information - 
World Data Center [38], where time series of only more than 100 years are considered.
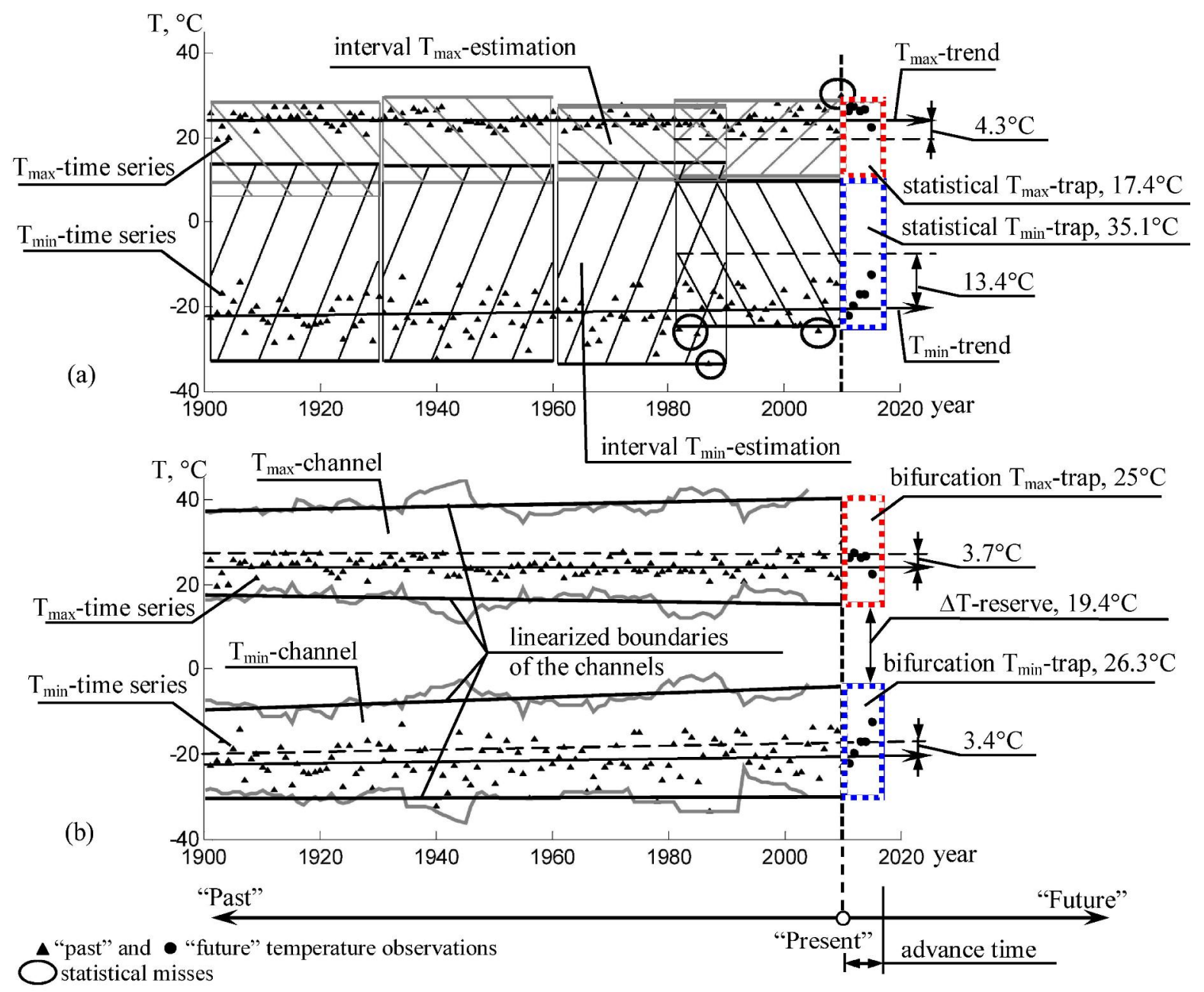

Fig. 5 - Example of the test made for statistical (a) and bifurcation (b) traps made for St.-Petersburg.

Let us illustrate typical differences between the results made from the traditional and proposed viewpoints. In this connection let us consider the temperature observations made in St.-Petersburg (WMO-code 26063 [38]) over 1900-2015 years as an example (Fig. 5, a, b). Let us imitate the estimations towards future based on the available data. Then, let 2010 year is accepted as the "present" (the corresponding "known" $\mathrm{T}_{\min }$ - and $\mathrm{T}_{\max }$-time series are denoted by black triangles); let the advance time is equal to 5 years $\left(\mathrm{T}_{\min }\right.$ - and $\mathrm{T}_{\max }$-time series in "future" are denoted by black circles). The traps are denoted by red and blue dotted outlines (for annual maximum and minimum correspondingly) on the right of the "present" and provide comparing two viewpoints.

The traditional viewpoint based on the ATVmodel supposes interval and trend estimations towards future $[19,22,29]$. The interval estimations suppose averaging over the accepted time window (one or several decades). For example, let us use the conventional 30-year division relatively 1961-1990 reference, including 1981-2010 years to fill the gap after 1990 year. So, in application to the considered issue, $\mathrm{T}_{\text {min }}$-evolution is determined for each window by $T_{\text {min av }} \pm 3 \sigma_{\text {min av }}$ (Fig. 1, a), where $T_{\text {min av }}$ and $\sigma_{\text {min av }}$ are a mean square value and a standard deviation correspondingly; $\quad \mathrm{T}_{\max }$-evolution is similarly determined. Then four pairs of the interval estimations occur to 2010 year (each pair is denoted by two-way hatching in Fig. 5, a).

Here it is necessary to note that the observed temperature extremes ( $\mathrm{T}_{\min ^{-}}$and $\mathrm{T}_{\max }$-time series) occur at various dates within a year; so, $\mathrm{T}_{\min }$ av and $\mathrm{T}_{\max \text { av }}$ deviate inevitably from these dates. Thus, interval estimations can lead to the following results (statistical $\mathrm{T}_{\max }$ and $\mathrm{T}_{\text {min }}$-traps in Fig. 5, a): sizes of the traps for annual maximum and minimum can be essentially different, and the extremes can be expected in future throughout whole temperature range without gaps. At the same time, these estimations can show incorrect result due to statistical misses (for example, misses over 19812010 years are denoted by ovals in Fig. 5, a). It indicates that the sampling includes heterogeneous data at least. In the context of the ATV-model such 
situations can not be excused; in the context of the HDS-model such situations are excused by extended deviations within $R L C$-ensembles consisting of three kinds of annual temperature patterns [27, 38].

Trend estimations seem to be more defined regarding the future events in comparison with the interval estimations $\left(\mathrm{T}_{\max }-\right.$ and $\mathrm{T}_{\min }$-trends denoted by black arrows in Fig. 5, a, b). However, such trend dissembles significant deviations from the basic tendency which are typical from time to time, especially, over half-century and more. For example, it occurs for evolution of $T_{\min }$-time series over the nineties of the XX-th century (Fig. 5, a): if the temperature jump has happened, then the basic tendency smoothes this fact. So, the nearest estimations towards future can include an additional asymmetry (for example, $\mathrm{T}_{\min }$-values over 20112015 years are more asymmetrical regarding $T_{\min }-$ trend in comparison with $\mathrm{T}_{\max }$-values over 20112015 years regarding $T_{\max }$-trend).

From the HDS-model, $\mathrm{T}_{\min }$-and $\mathrm{T}_{\max }$-channels are determined in order to identify tendency of $\mathrm{T}_{\text {min }}$-and $\mathrm{T}_{\max }$-evolution correspondingly (channels restricted by grey broken curves in Fig. 5, b). These channels show the upper and lower limits associated with $L$ and $R$-behaviors $\left(\mathrm{T}^{(\mathrm{L})}{ }_{\text {max av }}\right.$ and $\mathrm{T}^{(\mathrm{R})}{ }_{\max }$ av in the case of the temperature maximum; $\mathrm{T}^{(\mathrm{L})}{ }_{\min \text { av }}$ and $\mathrm{T}^{(\mathrm{R})}{ }_{\text {min av }}$ in the case of the temperature minimum) which were considered in the previous section. Per se, each of the channels combines the main advantages of the interval and trend estimations, namely: an interval description of the tendency observed in annual variation. The traps (hereafter bifurcation traps) are built as linearized boundaries of these channels (trends of the upper and lower limits denoted by black lines in Fig. 5, b).

Within the considered advance time ( 5 years), the centerlines of these traps (denoted by dashed lines) are noticeably closer to $T_{\min ^{-}}$and $T_{\max }$-observations than the ones determined for the statistical $\mathrm{T}_{\mathrm{min}^{-}}$and $\mathrm{T}_{\max }$-traps (Fig. 5, a). The bifurcation $\mathrm{T}_{\max }-$ and $\mathrm{T}_{\min ^{-}}$ traps are comparable in size and leave the significant temperature range, where the expected temperature extremes are absent $(\Delta \mathrm{T}$-reserve, Fig. $5, \mathrm{~b})$ in spite of the statistical $\mathrm{T}_{\min }$ - and $\mathrm{T}_{\max }$-traps (Fig. 5, a). The misses are absent and margins are less than the ones built from the ATV-model (the traps shown by Fig. 5, b in comparison with Fig. 5, a)

The results made on a set of the available meteorological data confirm the following items concerning the proposed method: homogeneity of samplings and, correspondingly, correctness of the statistical estimations can be provided; the logical linkage between evolution of several qualitatively different dynamical processes can be established; increase in quality of the seasonal forecasting due to decrease in margins of the $T_{\min }$-and $T_{\max }$-traps can be achieved. So, the novel statement on the seasonal forecasting could be promising for further research on the basis of the proposed method of bifurcation traps.

\section{CONCLUSION}

The results presented here and in [23, 24] represent a preview on the practice-oriented methods of the computer-based analytics of the temperature observations based on the contemporary scientific hypothesis, in accordance to which evolution of local climate dynamics is formed under the specific kind of feedbacks (hysteresis regulation with double synchronization). In this connection these methods are united by an attempt to find variants on how to apply the reconstructions of local climate behaviors from the corresponding conceptual model (HDSmodel) for real practical tasks.

As a result, the methods differ from the existing analogues in the combination of three important details: processing real observations over century and more; attracting the results of the bifurcation analysis; heightened time and space resolution of results (local daily means). This combination gives the following novel abilities: to reveal seasonal regularities of local climate dynamics with rather "weather resolution" in rather "climate scale" (here and in [24]); to derive constituents of regional (i.e. external) impacts on a local climate system by processing local temperature time series $[23,24]$.

Development of these abilities could reduce the deficiency of meteorological data thanks to additional useful information. Also, it could suggest other viewpoint on unsolved problems. In particular, it concerns the problem of the seasonal forecasting, were only advanced experimental works research forecasting of monthly means up to 1 year in future [34]. We demonstrate that the change of the conceptual model could potentially open a way to provide forecasting of local daily means up to more than 1 year in future.

The main disadvantages of the considered methods are connected with the necessary expert support to formalize analysis under the unusual conceptual models. Nevertheless, we believe that development of the methods should be continued. In what extent such research seems to be pressing? The answer could be the following: the more IT-tools are to research different viewpoints on coming climate changes, the more chances are to be ready to the nearest future.

\section{REFERENCES}

[1] C. L. Muller, L. Chapman, C. S. B. Grimmond, D. T. Young and X. Cai, "Sensors and the city: a review of urban meteorological networks," 
International Journal of Climatology, Vol. 33, pp. 1585-1600, 2013.

[2] S. Ribeiro, J. Caineta and A. C. Costa, "Review and discussion of homogenisation methods for climate data," Physics and Chemistry of the Earth, Parts A/B/C, vol. 94, pp. 167-179, 2016.

[3] S. Zilitinkevich, M. Kulmala, I. Esau and A. Baklanov, "Megacities - refining models to client environment," Bulletin WMO, Vol. 64(1), pp. 20-22, 2015.

[4] Z. Zong-Ci, L. Yong and H. Jian-Bin, "A review on evaluation methods of climate modeling," Advances in Climate Change Research, Vol. 4, Issue 3, pp. 37-144, 2013.

[5] www.ipcc-data.org/sim/

[6] Yu. Kolokolov and A. Monovskaya, "Guesswork and reasonings on centennial evolution of surface air temperature in Russia. Part II: Is it possible to research both local peculiarities and regional tendencies from the bifurcation analysis viewpoint?," International Journal of Bifurcation and Chaos, Vol. 26, 1650071, 2016.

[7] E. Kjellstrom, L. Barring, G. Nikulin, C. Nilsson, G. Persson and G. Strandberg, "Production and use of regional climate model projections - A Swedish perspective on building climate services," Climate Services, Vol. 2-3, pp. 15-29, 2016.

[8] M. F. Cabre, S. Solman and M. Nunez, "Regional climate change scenarios over southern South America for future climate (2080-2099) usig the MM5 Model. Mean, interannual variability and uncertainties," Atmosfera, Vol. 29(1), pp. 35-60, 2016.

[9] https://rcmes.jpl.nasa.gov/content/tutorialsoverview

[10] I. Maric, M. Pucar and B. Kovacevic, "Reducing the impact of climate change by applying information technologies and measures for improving energy efficiency in urban planning," Energy and Buildings, Vol. 115, pp. 102-111, 2016.

[11] L. Georgeson, M. Maslin and M. Poessinouw, "Global disparity in the supply of commercial weather and climate information services," Science Advances, Vol. 3(5), e1602632, 2017.

[12] M. B. Soares, M. Alexander and S. Dessai, "Sectoral use of climate information in Europe: A synoptic overview," Climate Services, 2017, available:www.sciencedirect.com/science/articl e/pii/S2405880717300018?via\%3Dihub.

[13] T. N. Palmer, "More reliable forecasts with less precise computations: a fast-track route to cloud-resolved weather and climate simulators?" Philosophical Transactions of the Royal Society A, Vol. 372, 20130391, 2014.
[14] C. Buontempo, C. Hewitt, F.J. Doblas-Reyes and S. Dessai, "Climate service development, delivery and use in Europe at monthly to interannual timescales," Climate Risk Management, Vol. 6, pp.1-5, 2014.

[15] M.B. Soares and S. Dessai, "Exploring the use of seasonal climate forecasts in Europe through expert elicitation," Climate Risk Management, Vol. 10, pp. 8-16, 2015.

[16] J. Feng, $\mathrm{Z}$. $\mathrm{Wu}$ and G. Liu, "Fast multidimensional ensemble empirical mode decomposition using a data compression technique," Journal of Climate, Vol. 27, pp. 3492-3504, 2014.

[17] P. Huybers, K. A. McKinnon, A. Rhines and M. Tingley, "US daily temperatures: The meaning of extremes in the context of nonnormality," Journal of Climate, Vol. 27, pp. 7368-7384, 2014.

[18] Yu. Kolokolov and A. Monovskaya, "Guesswork and reasonings on centennial evolution of surface air temperature in Russia. Part IV: Towards economic estimations of climaterelated damages from the bifurcation analysis viewpoint?" International Journal of Bifurcation and Chaos, Vol. 26, 1630033, 2016.

[19] J. Blunden and D. S. Arndt, Eds., 2016: State of the Climate in 2015, Bulletin of American Meteorological Society, Vol. 97, Issue 8, S1S275, 2016.

[20] V. Lucarini and T. Bodai, "Edge states in the climate system: exploring global instabilities and critical transitions," Nonlinearity, Vol. 30, R32-R66, 2017.

[21] Yu. Kolokolov and A. Monovskaya, "Modified bifurcation diagrams to analyse the intermittency observed in local climate dynamics," in Proceedings of the $8^{\text {th }}$ IEEE International Conference on Intelligent Data Acquisition and Advanced Computing Systems: Technology and Applications, Warsaw, Poland, September 24-26, 2015, pp. 624-630.

[22] B. Kirtman, et al. Near-term Climate Change: Projections and Predictability. In: Climate Change 2013: The Physical Science Basis. Contribution of Working Group I to the Fifth Assessment Report of the Intergovernmental Panel on Climate Change. Chapter 11, pp. 953-1028, Cambridge Univ. Press, Cambridge, UK and NY, NY, USA, 2013.

[23] Yu. Kolokolov and A. Monovskaya, "Observations-based computational analytics on local climate dynamics: change-points," International Journal of Computing, Vol. 16, Issue 2, pp. 89-96, 2017. 
[24] Yu. Kolokolov and A. Monovskaya, "Observations-based computational analytics on local climate dynamics. Part 2: Seasonality," International Journal of Computing, Vol. 16, Issue 3, pp. 152-159, 2017.

[25] B. Glahn, "Determining an optimal decay factor for bias-correcting MOS temperature and dewpoint forecasts," Weather and Forecasting, Vol. 29, pp. 1076-1090, 2014.

[26] F. Uboldi and A. Trevisan, "Multiple-scale error growth in a convection-resolving model," Nonlinear Processes in Geophysics, Vol. 22, pp. 1-13, 2015.

[27] Yu. Kolokolov and A. Monovskaya, "Guesswork and reasonings on centennial evolution of surface air temperature in Russia. Part III: Where is the joint between norms and hazards from the bifurcation analysis viewpoint?," International Journal of Bifurcation and Chaos, Vol. 26, 1650122, 2016.

[28] Valuing Weather and Climate: Economic Assessment of Meteorological and Hydrological Services, 2015 (WMO-No. 1153, World Meteorological Organization, Geneva, Switzerland).

[29] T.F. Stocker, et al. (eds.) "IPCC 2013: Summary for policymakers," Climate Change 2013: The Physical Science Basis. Contribution of Working Group I to the Fifth Assessment Report of the Intergovernmental Panel on Climate Change (Cambridge University Press, Cambridge), Chapter SPM, pp. 3-29, 2013.

[30] https://weather.gc.ca/saisons/prob_e.html

[31] http://www.hko.gov.hk/wxinfo/season/season. $\mathrm{htm}$

[32] http://wmc.meteoinfo.ru/season

[33] http://effis.jrc.ec.europa.eu/applications/seaso nal-forecast/

[34] http://www.cpc.ncep.noaa.gov/products/predic tions/long range/two class.php

[35] L. Goddard, J.W. Hurrell, B.P. Kirtman, J. Murphy, T. Stockdale and C. Vera, "Two time scales for the price of one (almost)," Bulletin of American Meteorological Society, Vol. 93, pp.621-629, 2012.

[36] J.A. Dutton, R.P. James and J.D. Ross, "Calibration and combination of dynamical seasonal forecasts to enhance the value of predicted probabilities for managing risk", Climate Dynamics, Vol.40, pp. 3089-3105, 2013.

[37] S. Pezzulli, D. B. Stephenson and A. Hannachi, "The variability of seasonality," Journal of Climate, Vol. 18, pp. 71-88, 2005.

[38] www.meteo.ru

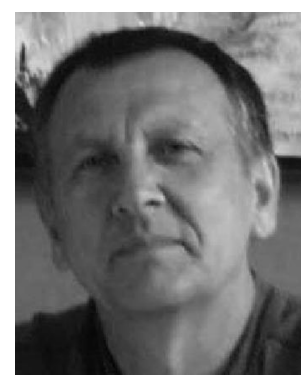

Yury Kolokolov received M.S. degree in 1972 (Tomsk Politechnical Institute, Russia), PhD degree in 1978 (Tomsk Politechnical Institute, Russia), Russian academic degree of Senior Doctor in 1990 (Tomsk University of Control and Radioelectronics, Russia). From

1993 to 2007 he was a full professor and Head of the Dept. of Design and technology of electronic systems (State Technical University of Orel, Russia); from 2007 to 2012 he was a full professor and Director of Institute of Control Systems and Information Technologies (UGRA State University, Khanty-Mansiysk, Russia); from 2012 to 2016 he was a chief researcher and a Head of Dept. of Applied Scientific Research (Russian Research Institute of Hydro meteorological Information - World Data Center, Obinsk, Russia). Currently he is a Head of scientific school (Orel State University, Russia). His scientific interests include theory, experiment and practice of nonlinear dynamics in the field of complex systems.

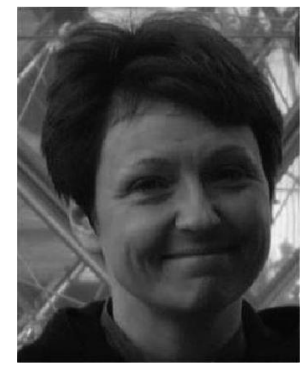

Anna Monovskaya received M.S. degree in 2001 (State Technical University of Orel, Russia), PhD degree in 2005 (State Technical University of Orel, Russia and University of Technology of Troyes, France), Russian academic degree of Senior Doctor in 2010. From 2012 to 2016 she was a chief researcher (Russian Research Institute of Hydro meteorological Information - World Data Center, Obinsk, Russia). Currently, she is a full professor and a Head of a Scientific-Educational Center (Orel State University, Russia). Her scientific interests include theory, experiment and practice of nonlinear dynamics in the field of complex systems. 